\title{
Diseño y construcción de un colorímetro para identificar el desgaste de fusibles comerciales usando sensores fotoeléctricos
}

\section{Design and construction of a colorimeter to identify the condition of commercial fuses using photoelectric sensors}

TORRES-FLORES, M. Azucena†, MARTÍNEZ-CARRILLO, Irma* y JUÁREZ-TOLEDO, Carlos

Universidad Autónoma del Estado de México, México.

ID 1 $1^{\mathrm{er}}$ Autor: M. Azucena, Torres-Flores / ORC ID: 0000-0003-4367-9431

ID $1^{\mathrm{er}}$ Coautor: Irma, Martínez-Carrillo / ORC ID: 0000-0002-7952-4418, Researcher ID Thomson: B-9264-2016, CVU CONACYT ID: 39914

ID $2^{\text {do }}$ Coautor: Carlos, Juárez-Toledo / ORC ID: 0000-0002-7440-3246, Researcher ID Thomson: C-1368-2016, CVU CONACYT ID: 39912

DOI: $10.35429 / J E E .2020 .12 .4 .16 .23$

Recibido Enero 20, 2020; Aceptado Junio 30, 2020

\section{Resumen}

El color es una propiedad que podemos observar gracias a nuestra percepción visual; sin embargo, es necesario de un instrumento para realizar la cuantificación en la escala RGB. El objetivo de este trabajo es diseñar y construir un colorímetro para identificar el grado de desgaste de las terminales de un fusible residencial convencional, que ha sido expuesto a su desgaste normal. El estudio se realiza por medio de la cuantificación de la reflexión de la luz en la escala RGB. El sensor desarrollado consta de:

- Una base cilíndrica elaborada en una impresora 3D, la cual permite aislar la luz externa y la introducción de la parte superior el casquillo del fusible.

- $\quad$ Un circuito electrónico conformado básicamente en un led emisor de luz RGB y una fotorresistencia.

- Un programa en Arduino que mide la reflexión del fusible en la escala RGB.

La implementación del sensor permite la identificación de los fusibles en mal estado y los cuales pueden provocar fallos en el sistema eléctrico, trabajos futuros estarán encaminados a la cuantificación del aumento del consumo energético debido al uso de fusibles en mal estado trasladados al espacio RGB.

Escala RGB, fusible residencial, impresión 3D

\begin{abstract}
Color is a property that we are able to observe thanks to our visual perception; however, an instrument is necessary to perform the quantification on the RGB scale. The purpose of this work is to design and build a colorimeter to identify the wear degree of a conventional residential fuse, the fuse has been exposed to normal wear. The study is carried out using the quantification of light reflection on the RGB scale. The developed sensor consists of:
\end{abstract}

A cylindrical base made on a 3D printer, which allows the external light to be isolated and the upper part of the fuse socket inserted.

An electronic circuit made up of RGB light emitting led and a photoresistor.

- An Arduino program that measures the reflection of the fuse on the RGB scale.

Implementing the sensor will allow the identification of fuses in poor condition, which can cause failures in the electrical system. Future work will be aimed at quantifying the increase of energy consumption due to the use of fuses in poor condition transferred in RGB space.

RGB scale, residential fuse, 3D printing

Citación: TORRES-FLORES, M. Azucena, MARTÍNEZ-CARRILLO, Irma y JUÁREZ-TOLEDO, Carlos. Diseño y construcción de un colorímetro para identificar el desgaste de fusibles comerciales usando sensores fotoeléctricos. Revista de Ingeniería Eléctrica. 2020. 4-12:16-23.

*Correspondencia al Autor (Correo Electrónico:imartinezca@uaemex.mx)

$\dagger$ Investigador contribuyendo como primer autor. 


\section{Introducción}

El uso eficiente de la energía eléctrica ha sido uno de los temas de interés mundial, donde la sociedad en general podemos colaborar activamente desde cualquiera de nuestras actividades cotidianas, como lo instituye en el Marco Político de Largo Plazo para la Eficiencia Energética que establece dentro de la línea transversal el realizar campañas de información, capacitación y mejores prácticas (SENER, 2016).

En relación con el uso eficiente de la energía, grandes medidas se han realizado para disminuir el consumo energético eléctrico innecesario, por ejemplo: por medio de un mantenimiento y supervisión del buen funcionamiento de circuitos, cambio de cables, mejora de equipos monitoreo de estado de fusibles, uso de interruptores diferenciales y cambio de dispositivos en mal estado.

El fusible residencial convencional es un dispositivo que tiene como principal función proteger las instalaciones eléctricas de sobrecargas o corto circuito, cuenta con una lámina de metal en la parte interior que al recibir un aumento de corriente este se abre y corta el paso de corriente evitando posibles daños a los equipos, esto ocurre dentro de un tiempo determinado (Eaton, 2018).

Existen diversos tipos de fusibles y están catalogados de acuerdo con la cantidad de energía que deben dejar pasar, con la finalidad de cubrir diferentes necesidades de protección como se indican en la tabla 1. En la figura 1 se muestran los fusibles más utilizados en la protección residencial.

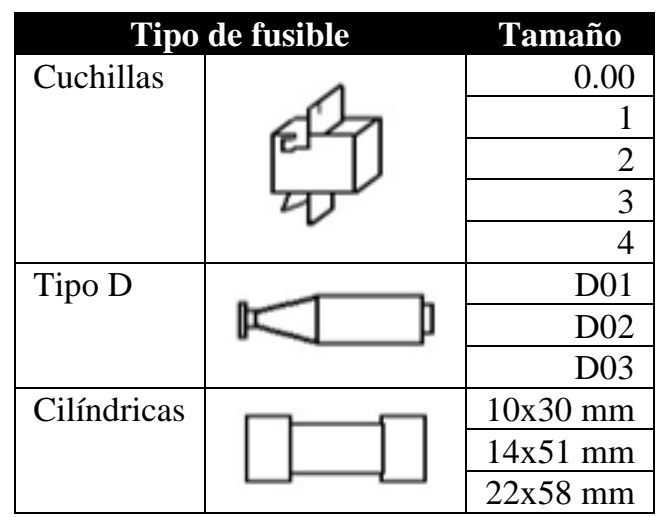

Tabla 1 Tipos de fusibles

Fuente: (García, 2008)

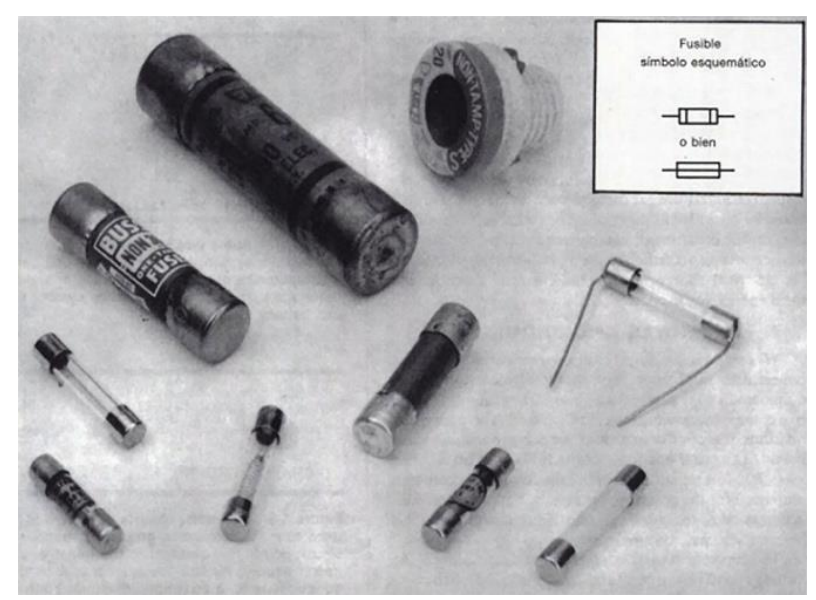

Figura 1 Aspecto de fusibles comunes Fuente: (Rinaldo, 1995)

Un fusible es el elemento más sensible de la instalación eléctrica, su función es que, en caso de sobrecarga, sea el componente que deba de cortar la corriente eléctrica, un ejemplo de su instalación se muestra en la figura 2.

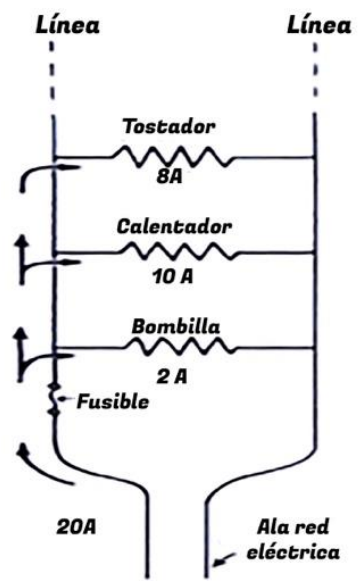

Figura 2 Diagrama eléctrico de una instalación domestica convencional

Fuente: (Hewitt, 2002)

Como se muestra en la fig. 2, el fusible se encuentra en el suministro de la red eléctrica en el cual al ocurrir un fallo en el sistema este abrirá para cortar el suministro de energía con lo que se protege el sistema. Sin embargo, se ha identificado que cuando un fusible permanece en una instalación trabajando en los limites para los que fue diseñado o bajo condiciones de humedad para los cuales no fue diseñado tiende a perder sus propiedades de baja resistencia eléctrica y pasa a ser una carga conectada en serie del sistema. Es por lo que en este trabajo se realiza un análisis del color de los fusibles comerciales residenciales para identificar el grado de desgaste de los fusibles. 
Un fusible se consigue a bajo precio, con una vida útil de aproximadamente 20 años sin sobrepasar sus capacidades nominales, no genera costos de mantenimiento (Guzmán, 2012).

Cuando el fusible se ha fundido es necesario remplazarlo por uno nuevo que tenga las mismas características y así asegurar la protección de los conductores, no se debe intentar componer la falla o puentear al cartucho para evitar incendios (Bastián et al., 2001). Por otro lado, están los fusibles renovables, que cuenta con una lámina insertada en la parte interior la cual se fundirá si recibe una sobrecarga, sin embargo, esta lamina pude ser sustituida por una nueva sin tener que cambiar el resto del fusible (Harper, 2004).

Esto es posible con la aplicación de la tecnología en un dispositivo que sea capaz de identifica la concentración de color en un elemento como el fusible, mismo que forma parte de las instalaciones domésticas, al conocer la concentración de color es posible saber si está funcionando de forma correcta.

El objetivo del presente trabajo es diseñar y construir un dispositivo (colorímetro) que sea capaz de reconocer e identificar el color de los casquillos de metal de los fusibles de cartucho tipo $h$ para conocer el estado y cambiarlos como mantenimiento preventivo.

\section{Diseño de sistema Autónomo de identificación de color}

El diseño y desarrollo de un sistema autónomo que identifique el color permitirá conocer la saturación de color que tienen los fusibles de cartucho tipo $\mathrm{H}$, con respecto a lo quemados que se encuentran.

El ahorro de energía es responsabilidad de todos y desde casa tenemos la oportunidad de hacerlo, actualmente existen diferentes sistemas inteligentes que trabajan gracias a la energía eléctrica y en favor de su ahorro.

En el presente trabajo se ha desarrollado el prototipo de un colorímetro que es capaz de identificar el tono y la saturación de color en fusibles de cartucho.
Su diseño está especialmente creado en SolidWorks, impreso en una impresora 3D y tomando en cuenta los fusibles de cartucho tipo $\mathrm{H}$, las principales características de la cámara son (ver figura 3):

- Tiene una profundidad para insertar el casquillo metálico del fusible de $3 \mathrm{~mm}$.

- Cuenta con una cámara 1 para albergar el led RGB.

- La cámara 2 contiene el sensor fotoeléctrico.

- Tiene una muesca o hueco entre las cámaras para dejar pasar la luz de reflexión del casquillo metálico.

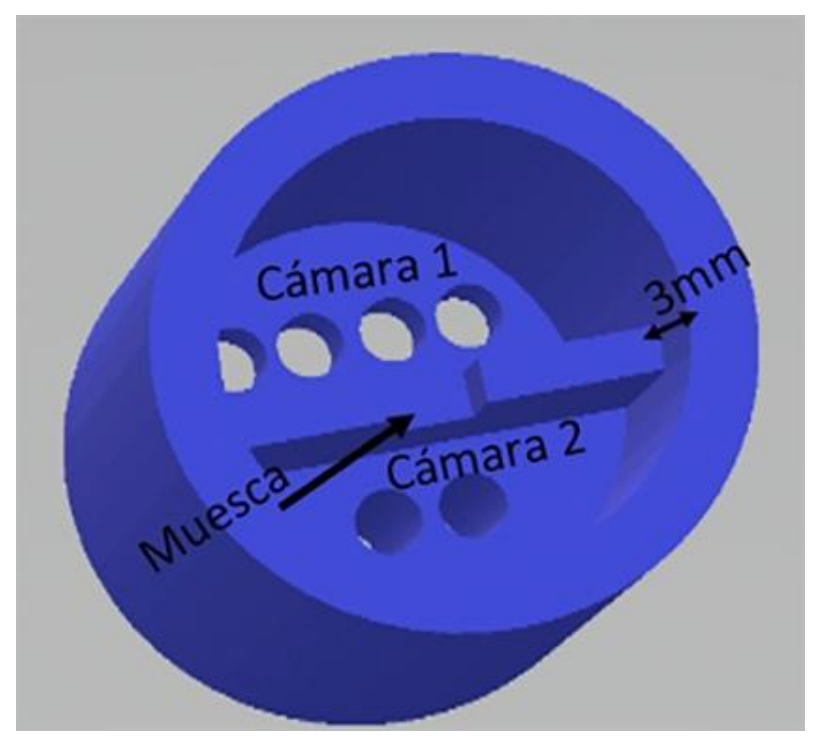

Figura 3 Base diseñada en SolidWorks e impresa en una impresora 3D para toma de lecturas del colorímetro Fuente: Elaboración propia

El sistema electrónico cuenta con dos botones de calibración del dispositivo y un botón actuador para iniciar el sistema de cuantificación del color. En las figuras 4 y 5 se muestra el diagrama físico y eléctrico respectivamente del circuito de control de color. 


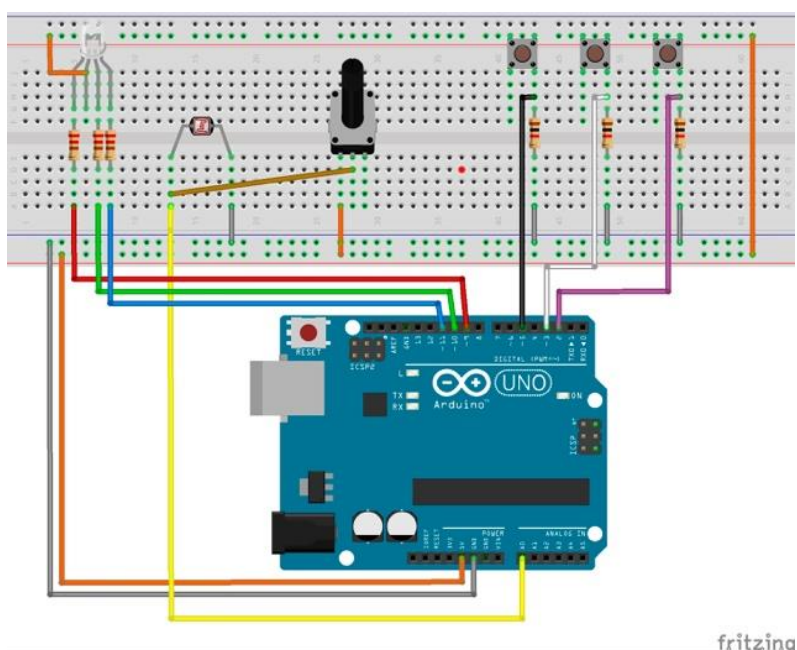

Figura 4 Diagrama físico

Fuente: Elaboración propia

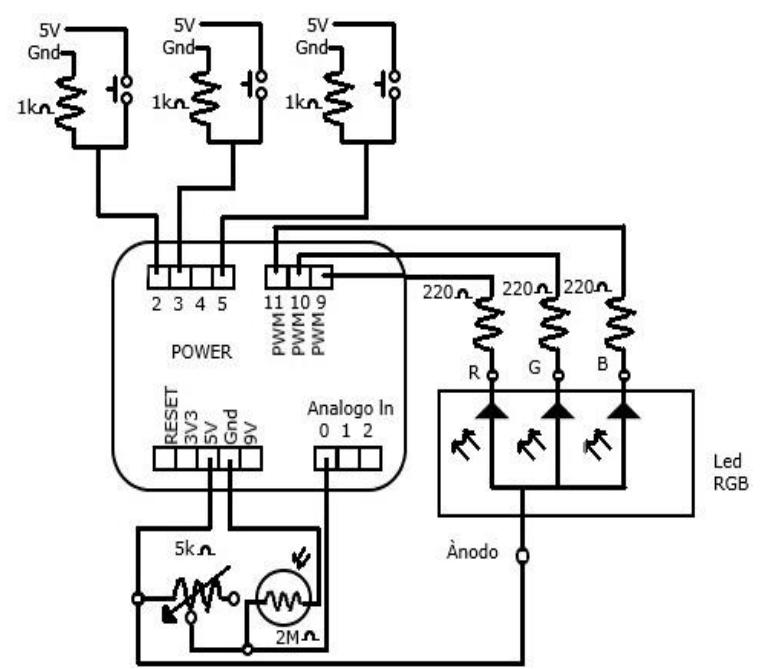

Figura 5 Diagrama eléctrico

Fuente: Elaboración propia

El sistema también cuenta con un potenciómetro de calibración de la fotorresistencia un led RGB protegido por tres resistencias.

El circuito electrónico básicamente consiste en emitir tres luces (Rojo, Verde y Azul) sincronizadas y tomar las lecturas de reflexión por medio de la fotorresistencia.

La programación en Arduino consiste en un bloque de calibración y tres bloques de accionamientos los cuales envían diferentes valores de referencia para obtener los colores Rojo $(255,0,0)$, Verde $(0,255,0)$ y Azul $(0,0,255)$, los cuales son monitoreados por la fotorresistencia, los valores del sensor son almacenados en memoria y mostrados en el monitor serie del software. La figura 6, muestra el diagrama de flujo para la programación del código para obtener las mediciones de color.

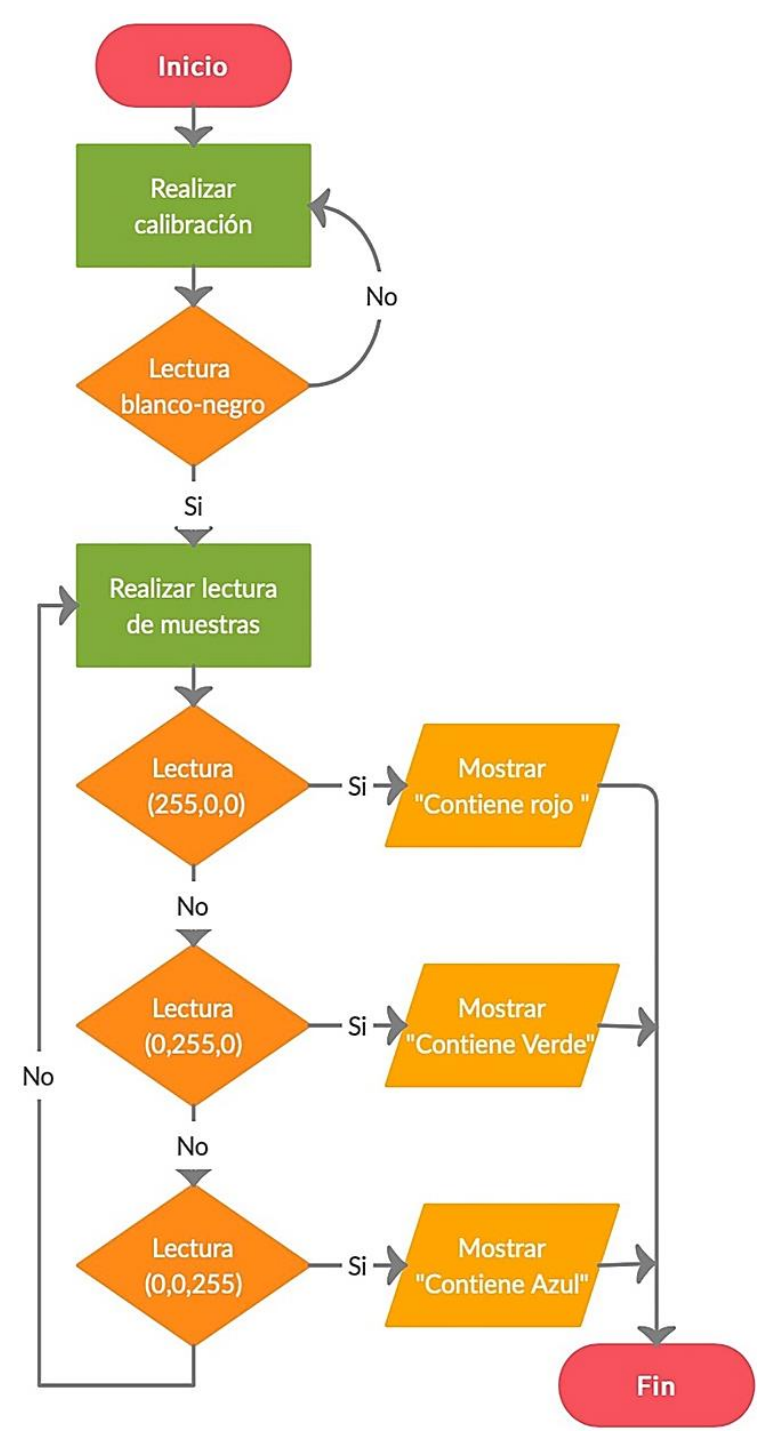

Figura 6 Diagrama de flujo de la programación de medición de color

Fuente: Elaboración propia

El prototipo desarrollado es de aproximadamente 14 centímetros y con un peso no mayor a 400 gramos, características que lo hacen portátil. En la figura 7 se muestra el prototipo desarrollado mostrando los botones de calibración, botón de accionamiento, cámara la cual contiene la fotorresistencia y el led RGB y el potenciómetro de calibración.

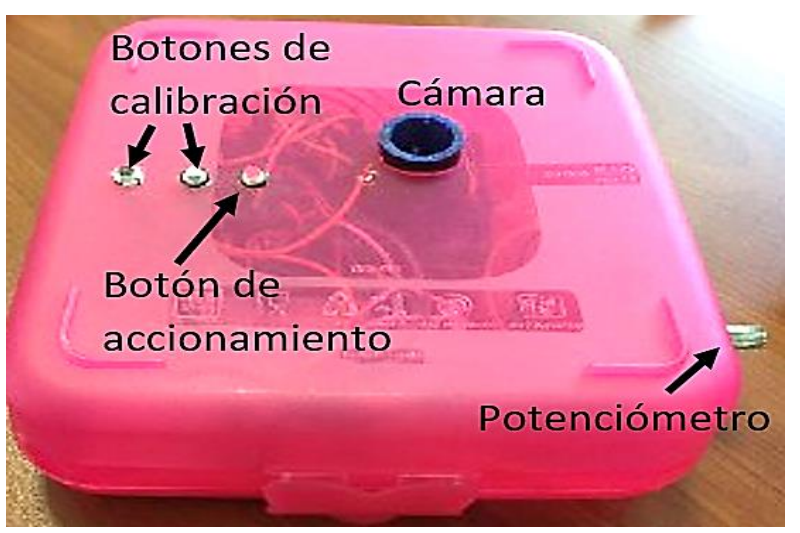

Figura 7 Prototipo de colorímetro encapsulado Fuente: Elaboración propia

TORRES-FLORES, M. Azucena, MARTÍNEZ-CARRILLO, Irma y JUÁREZ-TOLEDO, Carlos. Diseño y construcción de un colorímetro para identificar el desgaste de fusibles comerciales usando sensores fotoeléctricos. Revista de Ingeniería Eléctrica. 2020 
Durante la toma de lectura es necesario mantener conectado el prototipo por medio de la tarjeta Arduino a la computadora, la cual se describe de la siguiente manera:

- Abrir el software de Arduino comprobando el puerto COM de conexión.

- Conectar la tarjeta Arduino a la computadora, verificando el estado de parpadeo del led.

- Cargar el código en la tarjeta tomando en cuenta que no presente errores durante el proceso.

- Abrir el monitor serial para poder visualizar las lecturas cotejando que la velocidad de transmisión sea la adecuada.

- Calibrar el prototipo: colocar una tarjeta blanca sobre la superficie cilíndrica y oprimir el botón blanco, posteriormente pasar una tarjeta negra y oprimir el botón negro, con este proceso se almacena en memoria la máxima y mínima reflexión posible de un objeto, después de realizar la calibración, el dispositivo está listo para tomar la lectura y determinar el tono que predomina en la muestra.

- Tomar la lectura del fusible: colocar la parte metálica del fusible en la parte cilíndrica del prototipo y oprimir el botón de accionamiento

Una vez realizados los siguientes pasos, es posible visualizar los resultados en la consola de Arduino como se muestras en la figura 8.

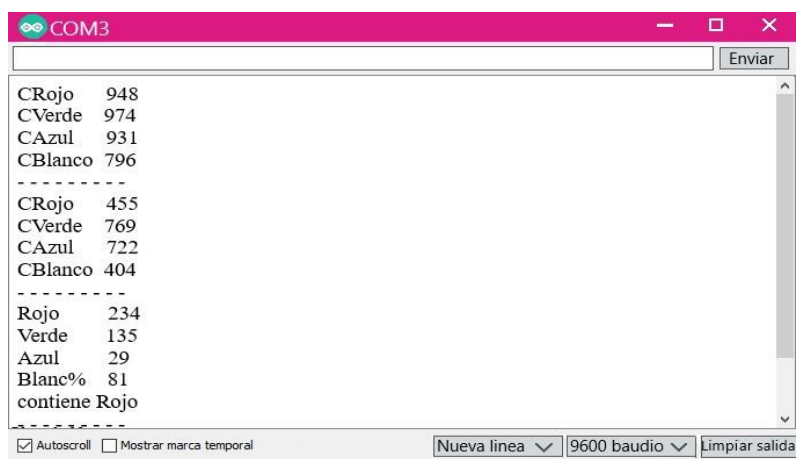

Figura 8 Monitor serial de Arduino y programa cargado en el dispositivo

Fuente: Elaboración propia

Después de tomar la lectura de la muestra es posible obtener la siguiente tabla 2 .

\begin{tabular}{|l|r|r|r|}
\hline Muestra & \multicolumn{1}{c}{ Rojo } & Verde & Azul \\
\hline $\mathrm{m} 1$ & 245 & 185 & 90 \\
\hline $\mathrm{m} 2$ & 264 & 109 & 104 \\
\hline $\mathrm{m} 3$ & 254 & 167 & 66 \\
\hline $\mathrm{m} 4$ & 193 & 105 & 38 \\
\hline $\mathrm{m} 5$ & 94 & 41 & 19 \\
\hline $\mathrm{m} 6$ & 74 & 31 & 16 \\
\hline $\mathrm{m} 7$ & 93 & 37 & 12 \\
\hline $\mathrm{m} 8$ & 59 & 19 & 0 \\
\hline
\end{tabular}

Tabla 2 Saturación de colores identificados Fuente: Elaboración propia

Se observa la saturación de color detectada por el colorímetro, con diferentes resultados respecto a su uso, en todas las muestras prevalece con mayor saturación el color rojo, el verde disminuye proporcionalmente con respecto al rojo y en la mayoría de los casos el azul hace lo mismo con respecto al color verde, también es posible observar que el color verde y azul en algunos casos tiene el mismo valor.

\section{Resultados}

Los fusibles utilizados para el desarrollo de este trabajo son fusibles de cartucho tipo $\mathrm{H}$ de 30 Amperes de baja tensión, para uso residencial. En la figura 9 se pueden apreciar los cuatro fusibles analizados, midiendo la parte superior e inferior de cada uno. En la figura 9 se puede observar a simple vista el desgaste de los fusibles, y es importante mencionar que los valores de los casquillos 1,2 corresponden a los fusibles con menor desgaste y los valores 7 y 8 son los de mayor desgaste.

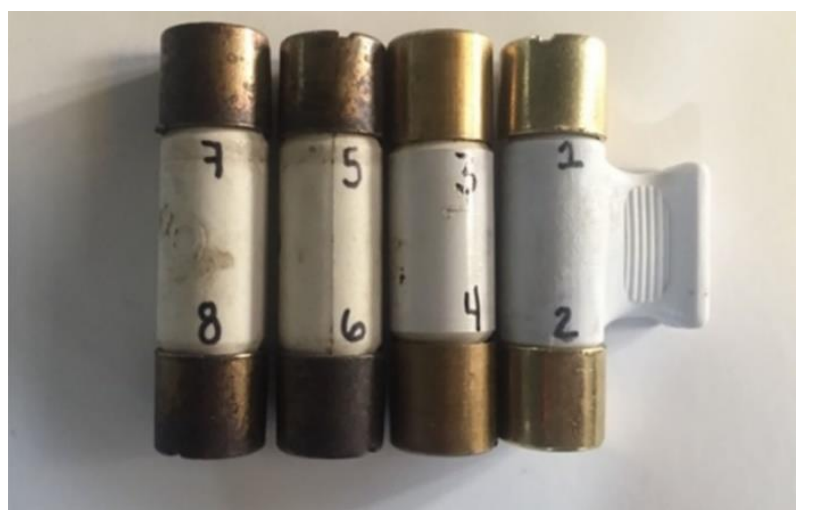

Figura 9 Muestras utilizadas en colorímetro Fuente: Elaboración propia

Además, es posible observar que mientras más quemada esta la muestra la saturación del color cambia. El gráfico 1 y el gráfico 2 muestran las mediciones obtenidas de color RGB. 


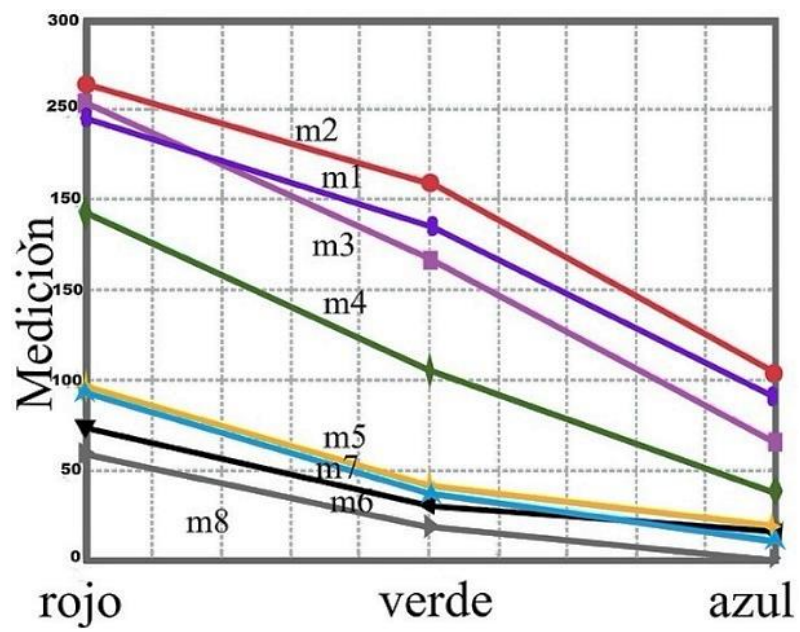

Gráfico 1 Medición en 2D de color RGB Fuente: Elaboración propia

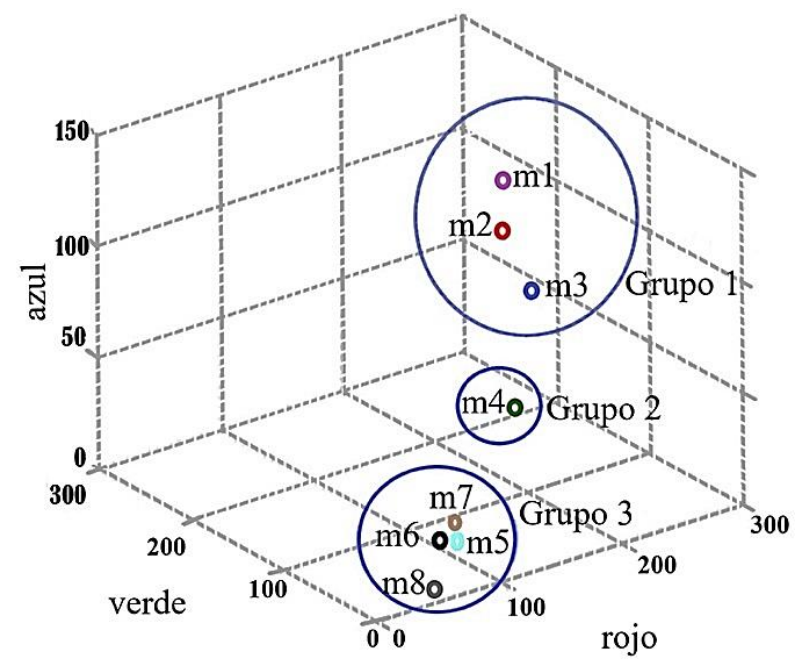

Gráfico 2 Comportamiento de muestras en espacio RGB Fuente: Elaboración propia

En los gráficos 1 y 2 se identifica tres grupos o clusters formados por:

- Grupo1 casquillos con menor desgaste, m1, $\mathrm{m} 2 \mathrm{y} \mathrm{m} 3$.

- Grupo 2 casquillos con mediano desgaste correspondiente al casquillo $\mathrm{m} 4$.

- Grupo 3 casquillos con mayor desgaste m5, $\mathrm{m} 6, \mathrm{~m} 7 \mathrm{y} \mathrm{m} 8$.

Para el caso de la muestra 1,2 y 3 , los niveles son más altos con respecto al resto de las muestras lo cual indica el bajo nivel de desgaste de los casquillos de este grupo.

En las muestras 3 y 4 a pesar de ser de un mismo fusible existe una variación significativa, lo cual indica que uno de los casquillos se encuentra más estrazado que su par.
A diferencia de las muestras 5, 6, 7 y 8 están notablemente alejadas del resto de los casquillos, los valores identificados se encuentran cercanos en esos casos, llegando a saturaciones más bajas de rojo, lo cual es congruente con el mayor tiempo que estuvieron en uso estos fusibles y además de que tuvieron que ser cambiados de la instalación por presentar un visible deterioro de las terminales.

\section{Conclusiones}

Con la finalidad de determinar el grado de desgaste de un fusible residencial, en el trabajo se desarrolló un prototipo que cuantifica el espectro de reflexión RGB de los fusibles residenciales. Para la implementación de su funcionamiento, se realizaron pruebas en diferentes fusibles con diferentes grados de desgaste.

El dispositivo está elaborado en ambiente Arduino y con procesamiento de datos en Matlab, es capaz de tomar lecturas de fusibles formado grupos "clusters" con índices de patrones congruentes. Trabajos futuros estarán encaminados a cuantificar la variación del gasto energético, así como del cambio de resistencia de los fusibles debidos a su desgaste.

\section{Agradecimientos}

A la Universidad Autónoma del Estado de México, UAP Tianguistenco, recursos PFCE 2020.

\section{Referencias}

Bastian, P., Eichler, W., Huber, F., Jaufmann, N., Manderla, J., Spielvogel, O., Springer, G., Stricker, F., Klaus, T. (2001). Electrotecnia, Akal.

Cervantes, J. R. (2000). Protección en Sistemas Eléctricos, Universidad Autónoma de Nuevo León.

Cruz, A. J., Cardona, G. J. \& Hernández, P. D. (2013). Aplicación electrónica para el ahorro de energía eléctrica utilizando una energía alternativa. Entramado, 9(2), 234-248.

Eaton. (2018). Fundamentos acerca de la protección contra sobrecorriente. Bussmann series, Powering Business Worldwide. 
García, T. J. (2008), Instalaciones Eléctricas En Media y Baja Tensión (6ta edición). Paraninfo.

Guzmán, M. G. (2012), Manual para coordinación de fusibles en la red de media tensión [Manual de bachillerato], Escuela de Ingeniería Eléctrica, Costa Rica.

Harper, E. (2004). Manual Práctico de Instalaciones Eléctricas, (2da Edición), Limusa.

Hewitt, P. G. (2002), Conceptual Physics, Novena edición, Pearson Educación.

Kamal Anoune., Mohsine Bouya., Abdelali Astito., Abdellatif Ben Abdellah. (2018). Sizing methods and optimization techniques for PVwind based hybrid renewable energy system: A review. Renewable and Sustainable Energy Reviews 93, 652-673.

Kamal T., Hassan S. Z., Li Hui., Mumtaz S., Khan L. (2016). Energy management and control of grid-connected wind/fuel cell/battery Hybrid Renewable Energy System. In: International Conference on Intelligent Systems Engineering (ICISE), Islamabad; p. 161-6.

Lagorse, J., Paire, D., Miraoui, A. (2010). A multi-agent system for energy management of distributed power sources. Renewable Energy 35, 174-182.

Luna-Rubio, R., Trejo-Perea, M., VargasVázquez, D., Ríos-Moreno, G. (2012). Optimal sizing of renewable hybrids energy systems: a review of methodologies. Solar Energy 86, 1077-1088.

Ma, T., Yang, H., Lu, L. (2014). Solar photovoltaic system modeling and performance prediction. Renewable and Sustainable Energy Reviews 3, 304-315.

Morales, R. D. \& Alvarado, L. E. (2014). Análisis del consumo de energía eléctrica domiciliaria en Tampico, Tamaulipas. Ciencia UAT, 8(2), 62-67.

Onar, O., Uzunoglu, M., Alam, M. (2008). Modeling, control and simulation of an autonomous wind turbine/photovoltaic/fuel cell/ultra-capacitor hybrid power system. Journal of Power Sources 185, 1273-1283.
Panapakidis, I., Sarafianos, D., Alexiadis, M. (2012). Comparative analysis of different gridindependent hybrid power generation system for a residential load. Renewable and Sustainable Energy Reviews 16, 551-563.

Pecen, R., Nayir, A. (2010). Design and implementation of a $12 \mathrm{~kW}$ Wind-Solar distributed power instrumentation system as an educational testbed for electrical engineering technology students. Proceedings of the international Symposium Modern Electric Power System (MEPS). Wroclaw, Polonia.

Rinaldo, P. (1995), Guía Internacional del Radioaficionado, Marcombo.

Secretaria de Energía (2016) Marco Político de Largo Plazo para la Eficiencia Energética.

Skoplaki, E., Palyvos, J.A. (2009). Operating temperature of photovoltaic modules: A survey of pertinent correlations. Renewable Energy 34, 23-29.

Stuchlý, J., Vramba, J., Misák, S. (2013). Monitoring of hybrid system using Labview. 13 th international conference on Environment and Electrical Engineering (EEEIC). Wroclaw, Polonia.

Uzunoglu, M., Onar, O., Alam, M. (2009). Modeling, control and simulation of a PV/FC/UC based hybrid power generation system for stand-alone applications. Renewable Energy 34, 509-520.

Vivas, F.J., De las Heras, A., Segura, F., Andújar, J.M. (2018). A review of energy management strategies for renewable hybrid energy systems with hydrogen backup. Renewable and Sustainable Energy Reviews 82, 126-155.

Wang, Ch., Chen, W., Shao, S., Chen, Z., Zhu, B., Li, H. (2011). Energy management of standalone hybrid PV system. Energy Procedia 12, 471-479.

Yang, H., Lu, L., Burnett, J. (2003). Weather data and probability analysis of hybrid photovoltaic-wind power generation systems in Hong Kong. Renewable Energy 28, 1813-1824. 
Zahraee S.M., Khalaji Assadi M., Saidur R. (2016). Application of Artificial Intelligence Methods for Hybrid Energy System Optimization. Renewable and Sustainable Energy Reviews 66, 617-630.

Zahran, M., Atia Y., Al-Hussain, A., El-Sayed, I. (2010). Proceedings of the 12th WSEAS International Conference on Automatic Control, Modelling \& Simulation. Wisconsin USA. 